\title{
WSC-07: Evolving the Web Services Challenge
}

\author{
M. Brian Blake \\ Georgetown University \\ Washington, DC 20057 \\ blakeb@cs.georgetown.edu
}

\author{
William K.W. Cheung \\ Hong Kong Baptist \\ University \\ Kowloon Tong, Hong Kong \\ williamhkbu@gmail.com
}

\author{
Michael C. Jaeger \\ Berlin University of \\ Technology \\ D-10587 Berlin, Germany \\ mcj@cs.tu-berlin.de
}

\author{
Andreas Wombacher \\ Ecole Polytechnical Fed de \\ Lausanne \\ Lausanne, Switzerland \\ andreas.wombacher@epfl.ch
}

\begin{abstract}
Service-oriented architecture (SOA) is an evolving architectural paradigm where businesses can expose their capabilities as modular, network-accessible software services. By decomposing capabilities into modular services, organizations can share their offerings at multiple levels of granularity while also creating unique access points for their peer organizations. The true impact of SOA will be realized when $3^{\text {rd }}$ party organizations can obtain a variety of services, on-demand, and create higher-order composite business processes. The Web Services Challenge (WSC) is a forum where academic and industry researchers can share experiences of developing tools that automate the integration of web services. In the third year (i.e. WSC-07) of the Web Services Challenge, software platforms will address several new composition challenges. Requests and results will be transmitted within SOAP messages. In addition, semantic representations will be both represented in the eXtensible Markup Language $(X M L)$ and in the Web Ontology Language (OWL). Finally, composite processes will have both sequential and concurrent branches.
\end{abstract}

\section{Introduction}

Succeeding the EEE-05 [3] [9] and the WSC-06 Challenges [2], the 2007 Web Services Challenge (WSC-07) [8]is the third year of this SOA venue [1] that looks to benchmark software applications that automatically manage web services. WSC-07, held at the IEEE Joint Conference on Electronic Commerce Technology (CEC 2007) and Enterprise Computing, ECommerce, and E-Services (EEE 2007) continues to provide a forum where researchers can collaborate on approaches, methods and algorithms in the domain of web service discovery and automated composition. This forum provides quantitative and qualitative evaluation results on the performance of participating matchmaking and automated composition software and facilitates the dissemination of results that advance this field.

Although in the third year, WSC-07 represents the fourth event of the matchmaking and composition challenge series. It extends the original criteria of the first two competitions which focused on service discovery and service composition based on the syntactic matching of WSDL part names. It also further extends the third competition in 2006 which provided a taxonomy of parameter types represented using the natural hierarchies that are captured using simple and complex types within XML documents.

WSC-07 is a new evolution of the challenge considering the adoption of semantically-defined data types. It has been the tradition of the WSC events to adhere to technology-independent approaches to semantics. In this way, the competition attempts to circumvent debates on representations, such as differences between approaches such as OWL-S [5] [4] and WSDL-S [10]. In 2006, the use of pure XMLbased semantics allowed for a bi-partisan approach. In 2007, we have evolved the challenge by mirroring the XML-based semantics with equivalent representations using an OWL ontology.

In earlier competitions, web service composition applications (i.e. participant entries) were constructed as stand-alone applications. In 2007, participating software must be implemented as web services. As such, requests and results of composition routines are transmitted as SOAP messages to and from the participating software applications. This natural progression in the WSCs will allow the competition itself to take place within a SOA environment.

Although in 2005, composition problems included the requirement to handle concurrent threads for composition routines, in 2006, the problem sets did not contain multiple branches. In WSC-07, concurrency is re-introduced into the composition problem sets. The combination of complex, workflow-based heuristics 
with semantically-rich representations will require participants to create new software that is both robust and efficient.

\section{Related Venues}

Although WSC is perhaps the first venue, other unique venues have been established to investigate the need for solutions to the service composition problem. The Semantic Web Services Challenge [6] is less of a competition and more of a challenge. Both business cases and solution applications are the focus of the venue. Participants are placed in a forum where they can incrementally and collaboratively learn from each other. While WSC venues are more driven by application, the SWS venues concentrate more on the environment. As such, the SWS venues place more focus on semantics where the WSC favors applied, short-term solutions.

Alternatively, the SOA Contest [7] held at the International Conference on Services Computing (SCC2006 and SCC2007) allows participants to openly choose the problems that best demonstrate their approach. The benefit of this venue is that participants can show the best approach for a particular domainspecific problem. In contrast, the WSC venue attempts to set a common problem where approaches can be evaluated side-by-side.

There is a unique niche for each forum and the composition of the results from all the venues will undoubtedly advance the state-of-the-art in serviceoriented computing.

\section{An Overview of Composition for WSC}

Within the WSC, the basic challenge asks to find an arbitrary service, $s_{x}$, from a set of available services, $S_{a}$, that matches an service request, $s_{r}$. A service request represents a service that expresses specific requirements. The challenge has to goal to simulate a software system that is capable of interpreting such requirements in order to identify a suitable service from $S_{a}$.

An arbitrary service, $s_{x}$, or service request, $s_{r}$, itself is comprised of two sets: One set of this service is $P_{x_{i}}$ which holds elements that represent the input parameters, and another set $P_{x_{o}}$ holds elements that represent the output parameters. An arbitrary parameter, $p_{y}$, denoted with small letters, represents an input or output parameter. A parameter is defined in a conceptual model of parameter types. This conceptual model is used to express relations among different parameter types.

Because the service model considers parameters of a service, the particular task required by the challenge is to identify matches between the parameters of the required service $S_{r}$ and those from a particular available service $s_{x}$. The relation,

$$
\forall p_{y} \in P_{r_{o}} \quad \exists p_{z} \mid \text { matching }\left(p_{y} \in P_{r_{o}}, p_{z} \in P_{x_{o}}\right),
$$

denotes that for the desired result of the service invocation, each particular required parameter is covered by a matching parameter from the available service. The relation,

$$
\forall p_{y} \in P_{x_{i}} \quad \exists p_{z} \mid \operatorname{matching}\left(p_{z} \in P_{x_{i}}, p_{y} \in P_{r_{i}}\right),
$$

denotes that for the invocation of the service, the service requester can offer all required parameters in order to ensure a proper invocation of the available service.

In order to cover a type hierarchy among the parameters, the challenge does not require that two parameters of each the required and available service not to be equal, but instead they must match. As such, the relation represented by matching $(a, b)$ can be defined by the compatibility of sub- and super-types as found in today's object-oriented languages. Thus, two parameters match when the two parameters refer to the same reference in the type hierarchy. Formally, two concepts match if the concept $a$ subsumes the concept $b$ according to the given type hierarchy. Otherwise the two parameters do not match.

In reality, the case might occur that no available service matches the required service. To cope with this case, the challenge asks also for the composition of a suitable service chain that is comprised of individual services from $S_{a}$. In this case, a list $S_{s}$ represents this chain forming is a subset from $S_{a}$. In addition, the services in $S_{s}$ have a common set of input and output parameters that are named $P_{s_{i}}$ and $P_{s_{o}}$. Then, regarding the inputs and outputs of the selected service the following conditions must be met:

$$
\begin{aligned}
& \forall p_{y} \in P_{r_{o}} \quad \exists p_{z} \mid \operatorname{matching}\left(p_{y} \in P_{r_{o}}, p_{z} \in P_{s_{o}}\right) \\
& \forall p_{y} \in P_{s_{i}} \quad \exists p_{z} \mid \operatorname{matching}\left(p_{z} \in P_{s_{i}}, p_{y} \in P_{r_{i}}\right)
\end{aligned}
$$

In addition, since the services form a chain, the inputs of services not leading the chain can be matched 
with the output of previously executed services. With respect to matching output parameters from services in the chain, only the required outputs a relevant. Unused output parameters do not interfere with a service invocation.

\subsection{Semantics}

For the semantic version of the challenge, a simplified version of semantic description of services is considered. Rather than using a full-fledged ontology based on an ontology language like OWL, a simplified type hierarchy defined in XML Schema is used to provide a common definition of domain concepts. In accordance, the required matching of parameter names is extended to the matching of parameter types defined by the XML Schema type hierarchy. WSC-07 includes an OWL mirror of this XML hierarchy to facilitate participants that prefer to parse OWL representations.

\subsection{Service Discovery}

With regards to service discovery, service predicates and requirements are provided and solution applications must gather a set of matching services based on their corresponding service interfaces. As a result, service discovery requires the following rules to successfully match the prescribed requirements to the parameters of candidate services:

- The types associated with the input parameters of the provided service must be subsumed by the types of the input parameters specified by the requester. If a service parameter type is not provided by the requester the successful execution cannot be guaranteed.

- The types associated with the output parameters of a candidate service must be equivalent or subsumed by the user-required output types.

\subsection{Service Composition}

WSC also considers the case that a single service does not match all input and output parameter types as requested. In this case, the service provider can compose services to fulfill the following constraints:

- The service requester must have all service inputs matched with their equivalent or subsuming types, and
- The service provider must match all required outputs with their equivalent or subsumed types. Otherwise the service does not fulfill the requirements of the requester by not delivering one or more required outputs.

In summary, the new matching challenge not only consider the string equivalence of parameter names, as this was the goal of the previous challenges, but also the contravariance of types to identify successful service matches. The considered type system is encoded using XML Schema. XML Schema is suitable for defining complex data types to establish a type hierarchy. Information expressed according to a type system using XML Schema can facilitate subsequent processing.

\section{Logistics of the Web Services Challenge}

WSC-07 has attracted international teams which come from universities and research organizations in countries including Austria, China, Germany, Singapore, and the United States. The organization of this event is divided into two phases: The first phase focuses on evaluating the technical viability of the methodologies proposed by the participating teams. A four-page technical description submitted from each team is peer-reviewed and included in the proceedings of the joint conferences (CEC/EEE2007). Once the reviewing and acceptance notification have completed, teams that successfully complete this first phase are asked to submit a version of their software for evaluation. Preliminary tests are conducted using the evaluation version to ensure the compatibility and applicability during the final competition. The main objective is to avoid in advance the potential format related problems that may otherwise occur when the competition takes place.

The second phase is the final competition which is scheduled for two days at CEC/EEE-07. On the first day, all the participating teams will present their approaches in a specialized session of the conference. On the same day, the participants are schedule times to install the latest version of their software on the evaluations services located onsite at the conference. On second day, the teams must execute their software using a customized data set prepared specifically for the competition.

Participating software is measured for performance during any indexing phases and during the actual composition routine. Composition results are evaluated against known solutions for correctness and completeness. In 2007 there will be multiple sets of 
correct answers which variable length chains. Applications will be judged with weighted scores based on the best solutions that they present.

The solution application with the best qualitative and quantitative scores when run again several datasets is awarded first place. The competition typically has a winner and several runner-ups. The winner in 2007 also wins a cash award.

\section{Acknowledgements}

The authors would like to acknowledge the efforts of Georgetown students David Cummings and Michael Fitzgerald Nowlan who facilitated the advertisement, review, and evaluation of the participating technical reports and software. Georgetown graduate student, John Adams, organized the travel logistics of the web service challenge. The authors also acknowledge Hong Kong Baptist graduate student, Kai-Kin Chan, for preparing the OWL representations. The Web Service Challenge has been extensively funded by the National Science Foundation under award number 0548514 and 0723990. The Hewlett Packard Corporation and Springer-Verlag have also supported an award to the winners of the competition.

\section{References}

[1] Blake, M.B., Cheung, W., and Wombacher, A. "Web Services Discovery and Composition Systems" International Journal of Web Services Research, Vol. 4, No. 1, pp iii - viii, January 2007

[2] Blake, M.B., Cheung, W., Jaeger, M.C., and Wombacher, A., "WSC-06: The Web Service Challenge", Joint Proceedings of the CEC/EEE 2006, San Francisco, California, USA, June 2006.

[3] Blake, M.B., Tsui, K.C., Cheung, W., "The EEE-05 Challenge: A New Web Service Discovery and Composition Competition" Proceedings of the IEEE International Conference on E-Technology, ECommerce, and E-Services (EEE-05), Hong Kong, March 2005.

[4] Fensel, D. and Bussler, C. "The Web Service Modeling Framework", Electronic Commerce: Research and Applications, 1(2): 113-137, 2002

[5] Martin, D. et al. "Bringing Semantics to Web Services: The OWL-S Approach", Proceedings of the First International Workshop on Semantic Web Services and Web Process Composition (SWSWPC-04), San Diego, CA, USA, July 2004.

[6] The Semantic Web Services Challenge (2007): http://sws-challenge.org/wiki/index.php/Main Page

[7] The Services Computing Contest (2007): http://iscc.servicescomputing.org/2007/

[8] The Web Services Challenge (2007). http://www.wschallenge.org/wsc07/

[9] The Web Services Challenge at the IEEE Conference on e-Business Engineering (ICEBE-05) (2007): http://www.comp.hkbu.edu.hk/simctr/wschallenge/

[10] WSDL-S $\underline{\text { http://www.w3.org/Submission/WSDL-S/ }}$ 